\title{
BMJ Open Mapping the national evidence on double burden of malnutrition in Ethiopia: a protocol of scoping review
}

\author{
Lemma Getacher (D) ,1,2 Beyene Wondafrash Ademe, ${ }^{2}$ Tefera Belachew (iD ${ }^{2}$
}

To cite: Getacher L, Ademe BW, Belachew T. Mapping the national evidence on double burden of malnutrition in Ethiopia: a protocol of scoping review. BMJ Open 2021;11:e050805. doi:10.1136/ bmjopen-2021-050805

- Prepublication history and additional supplemental material for this paper are available online. To view these files, please visit the journal online (http://dx.doi.org/10.1136/ bmjopen-2021-050805).

Received 03 March 2021 Accepted 20 0ctober 2021

Check for updates

(c) Author(s) (or their employer(s)) 2021. Re-use permitted under CC BY-NC. No commercial re-use. See rights and permissions. Published by BMJ.

${ }^{1}$ Department of Nutrition, School of Public Health, College of Medicine and Health Science, Debre Berhan University, Debre Berhan, Ethiopia

${ }^{2}$ Department of Nutrition and Dietetics, Faculty of Public Health, Institute of Health, Jimma University, Jimma, Ethiopia

Correspondence to

Lemma Getacher;

lemmagetacher@gmail.com

\section{ABSTRACT}

Introduction Currently, the double burden of malnutrition (DBM) is an emerging public health problem due to the inevitable consequences of nutritional transition. An increasing number of studies have reported the DBM in Ethiopian populations. However, organised and summarised national evidence is not yet available. Therefore, the main aim and research question of this scoping review is to map the national evidence on the DBM in the last decades in Ethiopia.

Methods This scoping review will be mapping the national evidence on the DBM in different population groups of Ethiopia including all ages and both sexes from 1 January 2021 to 30 September 2021. The Joanna Briggs Institute Reviewer's Manual will be used as the methodology of review for this review. The search strategy will be conducted using electronic databases such as PubMed, ScienceDirect, Cochrane Library, Wiley Online Library, Google Scholar, and grey literature sources and reference lists of key studies to identify studies appropriate for inclusion in the review. Two independent reviewers will be screened independently with all abstracts and full-text studies for inclusion. Data will be abstracted into tables and logically organised according to the items addressed in the specific research questions.

Ethics and dissemination The data will be used from publicly available secondary sources. Therefore, no ethical review and approval will be required for this review. Dissemination of results will be sought through peer-reviewed publications, conference presentations and stakeholder meetings. Regarding registration, this review protocol is registered on the figshare website on 28 February 2021, with the D0I number https:// doiorg/106084/m9figshare14131874.

\section{INTRODUCTION \\ Background}

The double burden of malnutrition (DBM) is the coexistence of undernutrition (stunting, wasting, underweight and micronutrient deficiency) and overnutrition (overweight, obesity and diet-related non-communicable diseases (NCDs)) at the individual, household, national and international levels simultaneously. Nowadays, it is an emerging public health problem that occurs due to the inevitable consequences of nutritional transition. ${ }^{12}$

\section{Strengths and limitations of this study}

- This scoping review will focus on the double burden of malnutrition (DBM) in a general population including children under 5 years of age, pregnant women, lactating mothers, adolescents and older populations.

- This scoping review is expected to provide an overview of the overall findings on DBM in Ethiopia and to pursue recommendations for future research.

- This is the first scoping review protocol on the topic of DBM for collating and summarising relevant published scientific evidence in a nationwide context.

- This scoping review will include studies that have either undernutrition or overnutrition and/or both undernutrition and overnutrition outcomes among the above-explained population groups in the country.

- This review will be limited to the English language with the interest of time and resources.

DBM is the two sides of a crisis that has vast health, environmental, economic, social and political implications, which affects every country of the world in its form. Worldwide, approximately one in three people and one in nine people are suffering from at least one form of undernutrition such as stunting, underweight, wasting, vitamin and mineral deficiencies, and overweight or obesity and diet-related NCDs, respectively. ${ }^{13}$

Approximately 462 million adults worldwide were underweight, 1.9 billion adults (>18 years) were either overweight or obese, 41 million children under the age of 5 years were overweight or obese, and 155 million children under 5 years of age were chronically undernourished. As a result, nearly $45 \%$ of deaths among children under 5 years of age are contributing to nutrition-related factors, majorly by undernutrition. Moreover, low-income and middle-income countries are now suffering from a simultaneous increase in childhood overweight. ${ }^{13-8}$

As various evidences show that DBM is one of the increased nutritional problems in all population groups as a life course problem. 
A study done in South Africa among mothers of primary school children showed that the prevalence of underweight, overweight and obesity was $2 \%, 27 \%$ and $42 \%$, respectively. ${ }^{9}$ Another study in Egyptian primary school children stated that the prevalences of thinness, overweight and obesity were $4.5 \%, 11 \%$ and $8.2 \%$, respectively. ${ }^{10}$ In a nutshell, several studies conducted in the global countries among children showed that DBM ranges from $5.6 \%$ to $49.8 \%{ }^{411-14}$ whereas in African countries DBM ranges from $15.7 \%$ to $54.2 \% .^{111516}$

Nutrition transition, a process of shifting from highly prevalent undernutrition to largely prevalent overnutrition, has become a major contributor to DBM in this world. This is related to the shifting of dietary patterns from high carbohydrate food to high fat food that has been found to largely coincide with rising rates of obesity and NCDs. ${ }^{17} 18$ Previously, the dominant consumed food types were carbohydrate type of foods, such as cereals and grains. Hence, foods made from cereals and grains, such as spaghetti, pasta, white bread, whole wheat bread, white rice, barley, oatmeal, waffle and so on, are high in carbohydrates. However, nowadays, due to the effect of globalisation and nutrition transition, most of the food types are dominated by fat such as frequently eating junk foods, fast food meals, cakes and pastries, sweets, processed meats, and sugary drinks. ${ }^{16-18}$

The key to prevention of diet-related diseases is to provide a better understanding of the shifts in dietary patterns and its drivers across populations. Worldwide, poor diet (either excess or inadequate intake) is the leading cause of mortality and morbidity exceeding the burdens attributable to many other major global health challenges. ${ }^{1} 1718$ Of the most important contributing factors behind the global pandemic of obesity, unhealthy diets composed of foods that are dense in energy, fat, sugar and salt are the most common. ${ }^{18}$

According to a systematic scoping review conducted in SSA, the major risk factors of childhood obesity were parental economic status, sex of the child, urban residence, consumption of highly refined/processed foods, snacks and sweetened beverages, parental level of education, age, low level of physical activity (sedentary lifestyle), attending private or public school, maternal obesity, screen time, birth weight, household size, stature, smoking, and loneliness. In addition, this study also identifies the common morbidities and comorbidities associated with childhood obesity/overweight. The most common morbidities and comorbidities associated with childhood obesity/overweight were hypertension (elevated blood pressure), dyslipidaemia, type 2 diabetes mellitus, iron deficiency anaemia, hypertriglyceridemia, vitamin D deficiency, parental diabetes status and low High-density lipoprotein (HDL) cholesterol. ${ }^{19}$

The world community aims to reduce malnutrition in all its forms, including overweight and obesity by 2030 through Sustainable Development Goals. The achievement of this objective is highly unlikely in the next few years before that date without a miracle, because its current achievement is very slow. ${ }^{2021}$

As the name indicates, the scoping review is an ideal approach/tool for exploring and describing the scope or coverage of a body of literature on a given topic (DBM in this review) and provides a clear indication of the volume of studies available and an overview, both broad and detailed, of its focus. ${ }^{22-24}$ The major indications of this scoping review are to identify the types of available evidence on DBM, clarify the key concepts/definitions of DBM, examine how research is conducted on DBM, identify key characteristics or factors related to DBM, as a precursor to a systematic review of DBM, and identify and analyse knowledge gaps in DBM in the country. ${ }^{225-27}$

\section{Study rationale}

A national overview of the evidence on DBM would provide an important basis for understanding how DBM was studied in Ethiopia. At a glance, in Ethiopia, the prevalence of DBM ranges from $1.99 \%$ to $57.7 \%$. More specifically, the prevalence of stunting ranges from $26.6 \%$ to $57.7 \%$, wasting ranges from $3.2 \%$ to $16.7 \%$, underweight ranges from $11.9 \%$ to $24.5 \%$, and overweight/obesity ranges from $1.99 \%$ to $42 \%{ }^{28-42}$ Even though little reduction in undernutrition exists, the increment of overnutrition among all populations of Ethiopia is another double burden and the summarised figure is not known.

The rationale of this review is to identify the existing knowledge gaps and recommend future research. The findings of this scoping review will rigorously provide a pattern, trend, summarise and conceptual framework for researching DBM. Ultimately such research findings will bring important insights for guiding policies, interventions and strategies aimed at reducing undernutrition, overnutrition and diet-related NCDs.

While several studies have been published on DBM, they are focused either on evidence from a specific population or on a subnational area. The national evidence on DBM has not been summarised so far on this issue. To the best of our knowledge, this scoping review will be the first to assess shreds of published on DBM nationally. Hence, now we propose a protocol of a scoping review aimed to summarise and collate the overall findings on the national evidence including all ages and sexes. We also hope that this work will help future researchers and bring an essential contribution to the field of nutrition.

Furthermore, DBM is a term used to identify a complex phenomenon (both undernutrition and overnutrition, several types of drivers of both) and the key concepts and definitions used in the literature require better clarification. Especially, it remains important to evaluate how research has been conducted on DBM in Ethiopia. Therefore, it has been proposed that conducting a scoping review on DBM would allow a better understanding of the patterns, trends, and determinants of DBM and provide a framework for further future research. 


\section{Study objectives}

A scoping review will be carried out to systematically map research findings investigating DBM in Ethiopia. Therefore, the main aim of this proposed scoping review is to summarise and map the national evidence on DBM duirng the last decades in Ethiopia.

\section{Research questions}

The research questions of this scoping review will take into consideration the population, concept and context (PCC). This review will address the following research questions:

\section{Main question}

- What is the national evidence on DBM in the different population groups of Ethiopia (from birth to old age) as a life course perspective?

\section{Subresearch questions}

- What is the prevalence of DBM reported in different population groups of Ethiopia?

- Is there a regional/subnational difference in double burden malnutrition in Ethiopia?

- What seems to be the trend of double burden malnutrition in Ethiopia?

- What are the main gaps in the existing kinds of literature and directions for further future research?

\section{METHODS}

\section{Protocol design}

Before designing the protocol of this scoping review, first, we have checked whether a scoping review on DBM is available or not through various databases and protocol registral websites. We checked it on protocol registration websites such as PROSPERO, Open Science Framework and figshare for any registered scoping reviews yet to be conducted. Consequently, there are no previously published scoping reviews on DBM in Ethiopia. Besides, this protocol was registered with the figshare registrar organisation with the DOI number https:// doiorg/106084/m9figshare14131874.

The protocol of this scoping review will be guided and developed using the scoping review tools of different scholars and the Joanna Briggs Institute. ${ }^{22} 234344$ Furthermore, the draft of the protocol was revised on receiving feedback from the research team. During protocol preparation, an article search will be done using keywords and index terms from 1 January 2021 to 30 September 2021.

After that, all eligible references will be imported to Mendeley desktop software V.1.19.5 and duplicates will be removed followed by title screening accordingly. Next, we will be performing an abstract screen for eligibility of the articles. Articles that will meet the priority criteria will be undergoing full review. Extraction of data will be taking place and analysis of the results will be done through the use of narrative analysis. The protocol will be guided by the Preferred Reporting Items for Systematic reviews and Meta-Analyses extension for Scoping Reviews (PRISMA-ScR) checklist guidelines. ${ }^{45}$

\section{Box 1 Inclusion criteria of the scoping review}

- To allow a better understanding of DBM patterns, trends and driver factors across populations, all age groups and both sexes will be included in the review.

- To get the appropriate trend and pattern of DBM, all dates of publication will be included.

- To get global audiences and readers, we include studies written in the English language.

- To get an appropriate knowledge base, any article type will be reviewed.

\section{Eligibility criteria}

To determine the eligibility criteria for this review, the PCC strategy/approach will be considered.

Population: The selected populations of this review will be all age groups as a life course approach (children under 5 years, school children, adolescents, pregnant women, breastfeeding women, adult population and older population) for both sexes.

Concept: The focus of this review will be on DBM (undernutrition and overnutrition that means overweight and obesity).

Context: The context of this review will be Ethiopia as a whole and its subnational states or regions.

\section{Inclusion criteria}

The inclusion criteria of the reviews are explained below in detail with the rationale behind each criterion (box 1).

\section{Exclusion criteria}

The exclusion criteria of the reviews are explained below in detail with the rationale behind each criterion (box 2).

\section{Search strategy}

Regarding the search strategy, published and unpublished (grey) literatures will be searched through advance search using several online databases such as PubMed, ScienceDirect, Cochrane Library, Wiley Online Library, Google Scholar and Google. The identified kinds of literatures will be screened based on their title, abstracts and key terms. The search terms included DBM, dual burden of malnutrition, nutrition transition, malnutrition, undernutrition, and overnutrition, micronutrient deficiencies and overweight and obesity, underweight and overweight and overweight, and obesity (online supplemental annex 1).

After that, a full assessment of papers that meet the inclusion and exclusion criteria will be retrieved. Reference lists

\section{Box 2 Exclusion criteria of the scoping review}

To address DBM in the best way, studies that have no outcomes in either undernutrition or overnutrition will be excluded.

- To avoid duplication of data, papers with no appropriate original data will be excluded.

- Since we are conducting a quantitative comparison and summary of studies, studies of a narrative style review with no secondary quantitative data will be excluded. 
of all included literature and excluded reviews will be additionally searched for any further relevant literature. To allow appraisal of possible publication bias, identified articles that have been published in languages other than English will be counted. No limitations will be set for the publication period. Mendeley desktop V.1.19.5 will be used as the reference management software to assist in the organisation and abstraction of data.

\section{Sources and selection of evidences}

As it is explained above in the inclusion criteria section, for the aim of this scoping review, the source of information can include any existing literature such as primary research studies, systematic reviews, meta-analyses, letters, guidelines, websites, blogs, commentaries and scoping reviews among others. To appropriately select the source of evidence, the protocol will be developed and based on six basic steps. These are identifying research questions, identifying relevant studies, selection of eligible studies, data abstraction and charting of results, data summary, and synthesis of. ${ }^{46}$ The detail of each step during the selection of evidences and sources is explained below.

\section{Step 1: Identifying research questions}

As explained above in the Research questions section, the main research question takes into account the PCC strategy/ approach.

\section{Step 2: Identifying relevant studies}

Based on the eligibility criteria, the relevant studies will be selected and searched from online databases using key terms and appropriate search strategies. During this time, there will be no timeframe restrictions for studies to get the available evidence.

\section{Step 3: Selection of eligible studies}

Before directly screening the studies, we will be conducting a calibration exercise with all reviewers to ensure reliability. Randomly, in our search, $10 \%$ of the citations as a sample will be screened independently by all reviewers. If the agreement is greater than $90 \%$, we will be beginning our article screening for review. Any conflicts which will occur in between will be resolved through peer discussion.

To identify the potentially eligible studies, the study selection method will be used as an elimination process based on title, abstract, key terms and full articles in ascending order by two independent reviewers. The selected results will be compared and consolidated through consensus between the two researchers. The final list of included articles will be further reviewed among the authors. Any disagreement on the final papers to be included will be discussed and an agreement will be arrived at through consensus by all authors. The PRISMA-ScR guidelines were used to present the number of included and excluded articles through a flowchart. ${ }^{44}$

\section{Data abstraction and charting of results}

Once the selection of studies has been completed, two researchers will independently conduct the data abstraction.
If any discrepancies have occurred between the two data extractors in the data abstraction process, they will be solved through further discussion. The abstracted data will be charted in a table including, but not limited to, authors, publication year, region, study design, sample size, population group, duration of the study period, outcomes measured and main findings. Then, this abstracted data will be used for the synthesis of findings in a narrative analysis form.

\section{Analysis of the evidence}

In this scoping review, we will be analysing the evidence descriptively and narratively to map the national evidence to DBM using narrative synthesis and content analysis.

\section{Presentation of the results}

The presentation of the results will be conducted using tables, charts and figures. The summary of the findings will provide an overview of the research conducted on DBM nationally. Data will be summarised both qualitatively (eg, overall summary of evidence) and quantitatively (eg, the proportion of subnational covered based on population). The key concepts and characteristics of the collected articles in the data extraction table will be summarised and finalised. To best capture the key characteristics of the published papers, the synthesised results may be further developed based on the findings of the scoping review.

\section{Ethics and dissemination}

Results from this scoping review will be published in a relevant peer-reviewed journal and presented at conferences. Because the sources of the data are based on the collection of publicly available data and due to the nature of the study, ethics approval is not required for this study.

\section{Patient and public involvement}

There are no participants (patients or public) who will be participating in this scoping review.

Contributors LG developed the protocol, research questions and methodology of the review. TB and BWA contributed to offering invaluable insight, comments, refinement and editing of the protocol. Besides, all authors are involved in the process of conception, preparation and approval of the final protocol of the manuscript.

Funding The authors have not declared a specific grant for this research from any funding agency in the public, commercial or not-for-profit sectors.

Competing interests None declared.

Patient consent for publication Not applicable.

Provenance and peer review Not commissioned; externally peer reviewed.

Data availability statement Data are available upon reasonable request.

Supplemental material This content has been supplied by the author(s). It has not been vetted by BMJ Publishing Group Limited (BMJ) and may not have been peer-reviewed. Any opinions or recommendations discussed are solely those of the author(s) and are not endorsed by BMJ. BMJ disclaims all liability and responsibility arising from any reliance placed on the content. Where the content includes any translated material, BMJ does not warrant the accuracy and reliability of the translations (including but not limited to local regulations, clinical guidelines, terminology, drug names and drug dosages), and is not responsible for any error and/or omissions arising from translation and adaptation or otherwise.

Open access This is an open access article distributed in accordance with the Creative Commons Attribution Non Commercial (CC BY-NC 4.0) license, which permits others to distribute, remix, adapt, build upon this work non-commercially, 
and license their derivative works on different terms, provided the original work is properly cited, appropriate credit is given, any changes made indicated, and the use is non-commercial. See: http://creativecommons.org/licenses/by-nc/4.0/.

\section{ORCID iDs}

Lemma Getacher http://orcid.org/0000-0002-9237-117X

Tefera Belachew http://orcid.org/0000-0001-5455-9457

\section{REFERENCES}

1 Initiatives D, Report GN. Action on equity to end malnutrition. Bristol, UK, 2020. https://globalnutritionreport.org/reports/2020-globalnutrition-report/

2 WHO. Double-duty actions for nutrition policy brief. Geneva, Switzerland: Department of Nutrition for Health and Development. World Health Organization, 2017. https://apps.who.int/iris/bitstream/ handle/10665/255414/WHO-NMH-NHD-17.2-eng.pdf?ua=1

3 Afshin A, Sur PJ, Fay KA, et al. Health effects of dietary risks in 195 countries, 1990-2017: a systematic analysis for the global burden of disease study 2017. The Lancet 2019;393:1958-72.

4 UNICEF/WHO/World Bank Group. Levels and trends in child malnutrition: key findings of the 2020 edition, 2020. Available: https:// data.unicef.org/wp-content/uploads/2020/03/Joint-MalnutritionEstimates-2020-brochure.pdf

5 WHO. Obesity and overweight factsheet No. 311, 2014. Available: https://www.mgwater.com/articles/WHO/Obesity and overweight WHO Fact Sheet No 311.pdf [Accessed 27 Jul 2020].

6 UN. Reduce child mortality. we can end poverty, factsheet. United nation, 2016. Available: https://www.un.org/millenniumgoals/pdf/ Goal_4_fs.pdf [Accessed 27 Jul 2021].

7 Vollmer S, Harttgen K, Kupka R, et al. Levels and trends of childhood undernutrition by wealth and education according to a composite index of anthropometric failure: evidence from 146 demographic and health surveys from 39 countries. BMJ Glob Health 2017;2:e000206-8.

8 Di Cesare M, Bentham J, Stevens GA. Trends in adult body-mass index in 200 countries from 1975 to 2014: a pooled analysis of 1698 population-based measurement studies with 19.2 million participants. Lancet 2016;387:96

9 Modjadji P. Socio-Demographic determinants of overweight and obesity among mothers of primary school children living in a rural health and demographic surveillance system site, South Africa. Open Public Health J 2020;13:518-28.

10 El-fatah NKA, Abu-elenin MM. Prevalence of stunting, overweight and obesity among Egyptian primary school children in Behera Governorate. Food and Public Health 2019;9.

11 Local Burden of Disease Child Growth Failure Collaborators. Mapping child growth failure across low- and middle-income countries. Nature 2020;577:231-4.

12 Giao H, Le An P, Truong Vien N, et al. Stunting and overweight among 12-24-month-old children receiving vaccination in $\mathrm{Ho}$ Chi Minh City, Vietnam. Biomed Res Int 2019;2019:1-7.

13 Tanwi TS, Chakrabarty S, Hasanuzzaman S. Double burden of malnutrition among ever-married women in Bangladesh: a pooled analysis. BMC Womens Health 2019;19:2-9.

14 Salehiniya $\mathrm{H}$, Yazdani $\mathrm{K}$, Barekati $\mathrm{H}$, et al. The prevalence of overweight and obesity in children under 5 years in Tehran, Iran, in 2012: a population-based study. Res Cardiovasc Med 2016:5:1-5.

15 khalil N, Megahed A, Ellahony D. Overweight and obesity among preschool children attending a rural family health unit in Menoufia governorate. EFMJ 2020;4:36-50.

16 Poda GG, Hsu C-Y, Chao JC-J. Factors associated with malnutrition among children $<5$ years old in Burkina Faso: evidence from the demographic and health surveys IV 2010. Int J Qual Health Care 2017;29:901-908.

17 Popkin BM. Nutrition in transition: the changing global nutrition challenge. Asia Pac J Clin Nutr 2001;10 Suppl:S13-8.

18 Popkin BM, Adair LS, Ng SW. Global nutrition transition and the pandemic of obesity in developing countries. Nutr Rev 2012;70:3-21.

19 Danquah FI, Ansu-Mensah M, Bawontuo V, et al. Risk factors and morbidities associated with childhood obesity in sub-Saharan Africa: a systematic scoping review. BMC Nutr 2020:6:1-14.

20 IFPRI. Global nutrition report 2015: actions and accountability to advance nutrition \& sustainable development. Washington, DC, 2015. https://www.ifpri.org/publication/global-nutrition-report-2015

21 Kraemer K. "Double burden of malnutrition": reexamining the coexistence of undernutrition and overweight among women in India In: Sight and life. Vol 32, 2018. https://sightandlife.org/wp-content/ uploads/2018/12/SALMZ_0218-1.pdf
22 Peters MDJ, Godfrey CM, Khalil H, et al. Guidance for conducting systematic scoping reviews. Int J Evid Based Healthc 2015;13:141-6.

$23 \mathrm{JBI}$. Supporting document for the Joanna Briggs Institute. levels of evidence and grades of recommendation. Adelaide, Australia: Joanna Briggs Institute (JBI), 2014. https://nursing.Isuhsc.edu/JBI/ docs/LevelsEvidence/SupportingDocuments.pdf

24 Munn Z, Peters MDJ, Stern C, et al. Systematic review or scoping review? guidance for authors when choosing between a systematic or scoping review approach. BMC Med Res Methodol 2018;18:143.

25 Khalil H, Peters MD, Tricco AC, et al. Conducting high quality scoping reviews-challenges and solutions. J Clin Epidemiol 2021;130:156-60. doi:10.1016/j.jclinepi.2020.10.009

26 Pham MT, Rajić A, Greig JD, et al. A scoping review of scoping reviews: advancing the approach and enhancing the consistency. Res Synth Methods 2014;5:371-85.

27 Tricco AC, Lillie E, Zarin W, et al. A scoping review on the conduct and reporting of scoping reviews. BMC Med Res Methodol 2016;16:15-10. doi:10.1186/s12874-016-0116-4

28 Fentahun W, Wubshet M, Tariku A. Undernutrition and associated factors among children aged 6-59 months in East Belesa district, Northwest Ethiopia: a community based cross-sectional study. BMC Public Health 2016;16:506.

29 Gelu A, Edris M, Derso T, et al. Undernutrition and associated factors among children aged 6-59 months living in slum areas of Gondar City, Northwest Ethiopia: a cross-sectional study. Pediatric Health Med Ther 2018:9:81-8.

30 Farah AM, Nour TY, Endris BS, et al. Concurrence of stunting and overweight/obesity among children: evidence from Ethiopia. PLoS One 2021;16:e0245456. doi:10.1371/journal.pone.0245456

31 Worku BN, Abessa TG, Wondafrash M, et al. The relationship of undernutrition/psychosocial factors and developmental outcomes of children in extreme poverty in Ethiopia. BMC Pediatr 2018;18:45.

32 Abebe Z, Zelalem Anlay D, Biadgo B, et al. High prevalence of undernutrition among children in Gondar town, Northwest Ethiopia: a community-based cross-sectional study. Int J Pediatr 2017;2017:5367070.

33 Abate $\mathrm{KH}$, Belachew T. Chronic malnutrition among under five children of Ethiopia may not be economic. a systematic review and meta-analysis. Ethiop J Health Sci 2019;29:265-77.

34 Medhin G, Hanlon C, Dewey M, et al. Prevalence and predictors of undernutrition among infants aged six and twelve months in Butajira Ethiopia: the P-MaMiE birth cohort. BMC Public Health 2010;10:27.

35 Egata G, Berhane Y, Worku A. Predictors of acute undernutrition among children aged 6 to 36 months in east rural Ethiopia: a community based nested case - control study. BMC Pediatr 2014;14:91.

36 Workie SB, Mekonen T, Fekadu W, et al. Level of undernutrition and its determinants among children aged 12-59 months in Wolaita district, Ethiopia. Pediatric Health Med Ther 2020;11:109-17.

37 Girma A, Woldie H, Mekonnen FA, et al. Undernutrition and associated factors among urban children aged 24-59 months in Northwest Ethiopia: a community based cross sectional study. BMC Pediatr 2019;19:214.

38 Zeray A, Kibret GD, Leshargie CT. Prevalence and associated factors of undernutrition among under-five children from model and non-model households in East Gojjam zone, Northwest Ethiopia: a comparative cross-sectional study. BMC Nutr 2019;5:27.

39 Delbiso TD, Rodriguez-Llanes JM, Donneau A-F, et al. Drought, conflict and children's undernutrition in Ethiopia 2000-2013: a metaanalysis. Bull World Health Organ 2017;95:94-102.

40 Jemal Z, Hassen K, Wakayo T. Household food insecurity and its association with nutritional status among preschool children in Gambella town, Western Ethiopia. J Nutr Food Sci 2016;06.

41 Desalegn BB, Kinfe E, Fikre K. Stunting and its associated factors in under five years old children: the case of Hawassa university technology villages, southern Ethiopia. IOSR J Environ Sci Toxicol Food Technol 2016;10 https://pdfs.semanticscholar.org/2e25/65d5 a96c13e63ccfe374594909e075123234.pdf

42 Tekile AK, Woya AA, Basha GW. Prevalence of malnutrition and associated factors among under-five children in Ethiopia: evidence from the 2016 Ethiopia demographic and health survey. BMC Res Notes 2019;12:391.

43 Levac D, Colquhoun H, O'Brien KK. Scoping studies: advancing the methodology. Implementation Sci 2010:5:1-9.

44 Arksey H, O'Malley L. Scoping studies: towards a methodological framework. Int J Soc Res Methodol 2005;8:19-32.

45 Tricco AC, Lillie E, Zarin W, et al. PRISMA extension for scoping reviews (PRISMA-ScR): checklist and explanation. Ann Intern Med 2018;169:467-73.

46 Aromataris E, Munn Z. JBI manual for evidence synthesis. Joanna Briggs Institute (JBI), 2020: 1-487. 